Pacific Journal of Mathematic 


\title{
A CONGRUENCE THEOREM FOR ASYMMETRIC TREES
}

\section{JAROSLAV NEŠETŘIL}

\begin{abstract}
The question is studied how a given tree is determined by the collection of its asymmetric subtrees. The results are analogous other partial answers to the Ulam-Kelly conjecture.
\end{abstract}

In [1], [2], [4], [5] several theorems are proved concerning the following conjecture posed by P. J. Kelly [4]: If $G$ and $H$ are two graphs with $p$ vertices $v_{i}$ and $u_{i}$ respectively $(p \geqq 3)$ such that for all $i: G-v_{i} \cong H-u_{i}$ then $G$ and $H$ are themselves isomorphic. In [4] it is shown that this conjecture is true when $G, H$ are trees. In [1], [2], [5] improvements of this result are obtained, namely, knowledge any of the following collections is sufficient to conclude $G \cong H$ providing $G, H$ are trees:

(1) all maximal proper subtrees [2]

(2) subtrees $T-v_{i}$ where $v_{i}$ is a peripheral vertex [1]

(3) non-isomorphic maximal subtrees [5].

Let $G(T)$ denote the automorphism group of a tree $T$. If $G(T)=$ \{identity\} then $T$ is called an asymmetric tree. Let $\mathfrak{X}$ denote the class of all asymmetric trees.

For a tree $T$ consider the set of all asymmetric proper subtrees of $T$. This set is naturally partially ordered by inclusion, denote by $A(T)$ the set of all maximal elements of this set, i.e. the set of all maximal asymmetric subtrees. (By subtree is meant proper subtree from now on.) Further denote by $\mathfrak{X}(T)$ the set of all isomorphism types of $A(T)$. (We denote by $[G]$ the isomorphism type of the graph $G$, hence $\mathfrak{U}(T)=\left\{\left[T^{\prime}\right]: T^{\prime} \in A(T)\right\}$.) We write $A(T) \cong A(S)$ for trees $T$ and $S$, if there is a one-to-one mapping $\varphi: A(T) \rightarrow A(S)$ such that $\varphi\left(T_{i}\right) \cong T_{i}$ for every $T_{i} \in A(T)$.

We write $\mathfrak{A}(T)=\mathfrak{U}(S)$ if the sets $\mathfrak{A}(T)$ and $\mathfrak{A}(S)$ are equal. We write $T_{i, j, k}$ for the tree consisting of three edge disjoint paths that start from a common point and have lengths $i, j, k$.

We will investigate the dependence of $[T]$ on $A(T)$ and $\mathfrak{V}(T)$.

It is obvious that not every tree $T$ will be determined by $A(T)$, since there are nonisomorhic trees with $A(T)=\varnothing$ (we do not include the trivial tree in the collections $A(T)$ and $\mathfrak{U}(T))$. But such trees are characterized by the following known result:

Proposition 0.1. We have $A(T) \neq \varnothing$ iff $T_{7}<T$, where $T_{7}=T_{1,2,3}$ with 7 vertices is the minimal asymmetric tree and $G<H$ means that $G$ is a proper full subgraph of $H$. 
Moreover, assuming $A(T) \neq \varnothing$, the minimal asymmetric subtrees: cover $T$, i.e., every edge of $T$ belong to some $T^{\prime} \in A(T)$, with the exception of the trees of one type. In view of this statement it would seem reasonable to conjecture that $\mathfrak{A}(T)$ and $T$ are in one-toone correspondence (up to isomorphism) providing $\mathfrak{U}(T) \neq \varnothing$. But this is not true, as is shown by the following class of examples:

Let $i(1), \cdots, i(n)$ be $n$ natural numbers. We denote by $T_{i(1), \ldots, i(n)}$ the subdivision of the $n$-star (i.e., $K_{1}+\bar{K}_{n}$, see [3]) obtained by inserting $i(k)-1$ points in the $k$ th edge. Obviously $\mathfrak{A}\left(T_{i(1) \ldots, i(n)}\right)=$ $\left\{\left[T_{1,2,3}\right]\right\}$ for every $n$ if $i(k) \leqq 3$ for $k=1, \cdots, n$. The situation cannot be saved by considering $A(T)$ rather that $\mathfrak{X}(T)$ since $A\left(T_{3,2,2}\right) \cong$ $A\left(T_{32,1,1}\right)$. The examples given here are not unique. We prove:

Main Theorem weaker form. Let $T, S$ be asymmetric trees. Then $A(T) \cong A(S) \Leftrightarrow S \cong T$

Main Theorem stronger form. Let $S, T$ be asymmetric trees. Then $\mathfrak{A}(T)=\mathfrak{A}(S) \Leftrightarrow T \cong S$, with the exception of the following two trees:

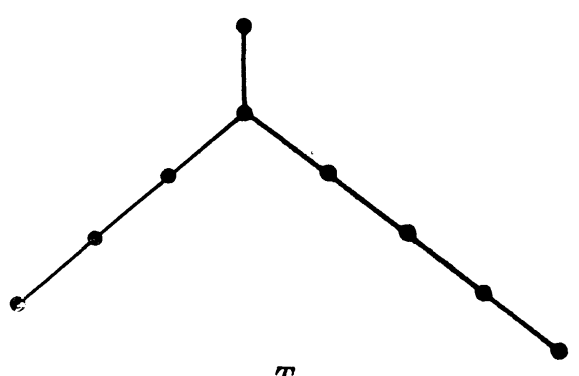

$T_{1,3,4}$

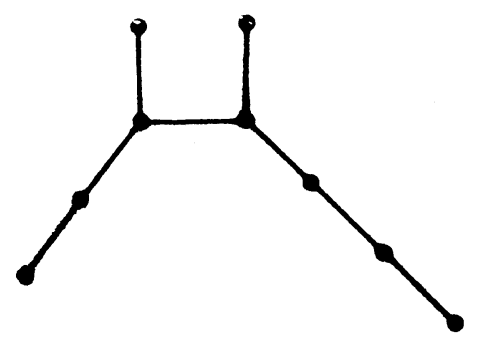

$T_{1}$

Since obviously $A\left(T_{1.34}\right) \not A\left(T_{1}\right)$ it is enough to prove the stronger form of the main theorem. In fact we prove this theorem in reformulation of the problem as a reconstruction of a tree (see Theorem 2.1).

The paper has two parts. In first of them we investigate the group of automorphisms of a tree in general and its connection to asymmetry (Corollary 1.2), in the second part we prove the main theorem (Theorem 2.1). The notions of the graph theory not defined here may be found in [3].

\section{The automorphism group of a tree.}

${ }^{1}$ I thank B. Manvel, who found independently the examples of exceptional trees. $T_{1,3,4}$ and $T_{1}$ and called my attention to them. 
Theorem 1.1. Let $T$ be a tree. Let $C_{2}(T)=\{f \in G(T): f \circ f=i d$.$\} .$ Then $C_{2}(T)$ generates $G(T)$.

Proof. Since every symmetric group is generated by transpositions and direct products and compositions see [3] preserve generators, the theorem follows.

THEOREM 1.1 has some interesting consequences:

CoROllary 1.1. Let $T$ be a symmetric tree (i.e., $G(T) \neq i d$. .). Then there is $f \in G(T), f \neq i d$. and $f \circ f=i d$.

This is clear by the above theorem. We remark that this is already false for unicyclic graphs, since there is a graph $X$ with $G(X) \cong C_{3}$ (the cyclic group of order 3 ), see [3] p. 169.

Corollary 1.2. Let $T$ be an asymmetric tree, $d(x, T)=1$. (By $d(x, T)$ we denote the degree of the point $x$ in the tree T.) Then $|G(T-x)| \leqq 2$ (i.e. the removing of an endpoint of an asymmetric tree gives rise to at most one symmetry).

Proof. Suppose for the contrary $|G(T-x)|>2$ for some $d(x, T)=1$. By Theorem 1.1 there are $f_{1}, f_{2} \in G(T-x)$ and $f_{1} \circ f_{1}=$ $f_{2} \circ f_{2}=i d ., f_{1} \neq f_{2}$. Let $[x, y] \in E(T)$, then necessarily $y \neq f_{1}(y) \neq$ $f_{2}(y) \neq y$. Let us distinguish two cases: (i) $T-x$ is a central tree (see [1]), $c$ is the only center of $T-x$. Let $W(c, y)$ be the path joining $y$ and $c$. Put $n_{i}=\min \left\{\rho(c, z) ; z \in W(c, y), f_{i}(z) \neq z\right\}, i=1,2$. $(\rho(c, z)$ is the distance between $c$ and $z$.) It can be proved easily that $n_{1}=n_{2}$ and that $f_{1}(z)=f_{2}(z)$ where $z \in W(c, y), \rho(z, c)=n_{1}$. But then $f_{1}=f_{2}$, for otherwise the number $n$, defined for $f_{1}^{-1} \circ f_{2}$ as $n_{1}$ was defined for $f_{1}$, would be greater than $n_{1}$. But $f_{1} \neq f_{2}$ by hypothesis. (ii) Let $T-x$ be bicentral. We can use the same argument as in (i) for the tree $(T-x)^{2}$, where for every $T$-bicentral tree the central tree $T^{\vee}$ is defined by: $V\left(T^{\vee}\right)=V(T) \cup\{c\}, c \in V(T)$ and $E\left(T^{\vee}\right)=$ $\left(E(T)-\left[c_{1}, c_{2}\right]\right) \cup\left[c, c_{1}\right] \cup\left[c, c_{2}\right]$, where $c_{1}, c_{2}$ are two centers of $T$.

REMARK. Corollary 1.2 gives a necessary condition for a tree $T$ to have an asymmetric extension to $|T|+1$ vertices, which is itself a tree. This condition is not sufficient.

2. Asymmetric congruence of trees. We are going to prove the main theorem. This will be done in Propositions 2.1-2.8. A difference between the proof presented here and the proofs used in [1], [2], [4] is that we know less about the structure of $\mathfrak{A}(T)$. Thus 
to prove that some basic parameters of $T$ are determined by $\mathfrak{X}(T)$ we need existence theorems.

Let $T \in \mathfrak{R}$ be fixed from now on.

Proposition 2.1. $\mathfrak{A}(T)=\varnothing$ iff $T \cong T_{7}$.

This follows from Proposition 0.1. Thus let $\mathfrak{X}(T) \neq \varnothing$ from now on.

Let $(T, x)$ be a rooted tree, by $G(T, x)$ we denote the group of all root-automorphisms, i.e., all automorphisms of $T$ which leave $x$ fixed. In an obvious sense we will speak about root-asymmetric tree, root-isomorphic trees $(T, x) \cong(S, y)$ and so on.

Let $T$ be a tree, the branch $S$ of $T$ at a point $x$ is every maximal subtree of $T$ which contains $x$ as an endpoint. Every branch at a center of $T$ is called limb.

To determine $|T|$ we prove the existence of $\left[T_{0}\right] \in \mathfrak{A}(T),\left|T_{0}\right|=$ $|T|-1$. We prove first:

Lemma 2.1. Let $\left(T, x_{0}\right)$ be a root-asymmetric tree. Then there is a vertex $x, x \neq x_{0}, d(x, T)=1$, such that $\left(T-x, x_{0}\right)$ is root-asymmetric.

Proof. For $|T|=2$ the statement obviously holds. Let the lemma hold for every $(S, y),|S|<n$. Let $\left(T, x_{0}\right)$ be a root-asymmetric tree, $|T|=n$. Define the relation $\prec$ on $V\left(x_{0}, T\right)=\left\{x ;\left[x, x_{0}\right] \in E(T)\right\}$ by: $x \prec y \Leftrightarrow$ there is an endpoint of $T_{y}$ and $\left(T_{y}-z, x_{0}\right) \cong\left(T_{x}, x_{0}\right)$. (Equivalently by Corollary 1.2: there is an endpoint $z \in V\left(T_{y}\right)$ and $f \in G(T-z)$ such that $f(y)=x, f(x)=y$.) Here $T_{y}$ denotes the branch of $T$ at $x_{0}$ containing $y$.

Let $x_{1}$ be a minimal vertex for the relation $\prec$. Then by the induction hypothesis there is $x \in T_{x_{1}}$ such that $\left(T_{x_{1}}-x, x_{0}\right)$ is rootasymmetric and by the definition of $\prec\left(T-x, x_{0}\right)$ is a root-asymmetric tree.

According to [1], a vertex $x$ of a tree $T$ is called peripheral if there is $y \in V(T)$ and $\rho(x, y)=\operatorname{diam} T$. The couple $x, y$ we call a peripheral couple.

Proposition 2.2. ( I ) Let $T$ be a central tree, then either ( i) $T \cong T_{1, k, m}, k+m$ odd or (ii) there is $\left[T_{i}\right] \in \mathfrak{N}(T), T_{i}$ central, $\left|T_{i}\right|=$ $|T|-1$.

(II) Let $T$ be bicentral, then either (i ) $T \cong T_{1, k \cdot m}, k+m$ even or (ii) there is $\left[T_{i}\right] \in \mathfrak{A}(T), T_{i}$ bicentral, $\left|T_{i}\right|=|T|-1$.

(III) For every $T$ there is $T_{i} \in A(T), T_{i}$ is a maximal subtree.

Proof I. Let $T$ be a central asymmetric tree with the center $c_{\text {. }}$ The following cases are possible: 
(a) for every peripheral vertex of $T$, there is a peripheral couple disjoint with it

(b) $T$ contains more than one peripheral couple and (a) does not hold

(c) $T$ contains exactly two peripheral vertices.

In the case (a) we can use Lemma 2.1 since each $T-x$ is a central tree and hence $(T-x, c)$ is a root-asymmetric tree if $T-x$ is an asymmetric tree. Suppose that (c) holds: Let $a, b$ be the only peripheral vertices of $T, a \in R_{1}, b \in R_{2}$ the only radial limbs of $T$ (i.e., branches at the center with a peripheral point). Let $\left|R_{1}\right| \leqq\left|R_{2}\right|$. If there are other limbs of $T$, then we can apply Lemma 2.1 to their union and find $x$ such that $[T-x] \in \mathfrak{A}(T)$. Thus let $R_{1}, R_{2}$ be the only branches of $T$ at $c$. The proof can be finished by choosing a convenient point $z \in W(a, b)$ (the unique path connecting $a$ and $b$ ), $d(z, T) \geqq 3$ and considering the union of all branches of $T$ at $z$ which contain neither $a$ nor $b$. Using Lemma 2.1 we get an asymmetric tree with the only exception $T \cong T_{1, k, m}, k+m$ odd.

The case (b) can be handled similarly.

(II) can be proved by use of the graph $T^{\curvearrowright}$ (see the proof of the Corollary 1.2).

(III) is obvious by (I) and (II), since $T_{1,2,3} \neq T_{1, k, m}$ implies that $T_{1, k m}$ contains a maximal subtree which is asymmetric.

REMARK. The Proposition 2.1. (III) was recently proved in a different context by J. Sheehan and J. A. Zimmer Jr. from the University of Waterloo.

Proposition 2.3. Let $\{k, m\} \neq\{3,4\}$. Then $T_{1, k, m}$ is reconstructible from $\mathfrak{X}\left(T_{1, k, m}\right)$. There is $\mathfrak{U}\left(T_{1,3,4}\right)=\mathfrak{U}\left(T_{1}\right)$ (see the Introduction) and there are no other such graphs.

Outline of proof. Obviously $\left[T^{\prime}\right] \in \mathfrak{A}\left(T_{1, k, m}\right)$ implies $T_{2,2,2} \not T^{\prime \prime}, T^{\prime}$ has only one vertex of degree $\geqq 3$, and further $\left|\mathcal{A}\left(T_{1, k, m}\right)\right| \leqq 2$. From these facts one can verify the statement by exhaustion of cases.

Proposition 2.4. (i) $T$ is central if diam $T^{\prime \prime}<$ diam $T$ for every bicentral subtree $T^{\prime}$ of $T$ (diam $T$ is the diameter of the tree $T$ ) (ii) $T$ is bicentral if diam $T^{\prime}<$ diam $T$ for every central subtree $T^{\prime}$ of $T$.

The proof is clear and is omitted.

By Propositions 2.2, 2.3, 2.4 we can determine from $\mathfrak{A}(T)$ whether 
$T$ is central or bicentral. Thus from now on let $T$ be central, $T \not$ $T_{1, k, m}, T \not T_{1}$. Let $c$ be the center of $T, r$ the radius of $T$, (see [3]). The case $T$ bicentral will be investigated later. The following lemma deals with a special kind of trees, one that has a radial limb which is a path (called a radial path).

\section{LEMMA 2.2. The following two statements are equivalent:}

(i) $T$ satisfies one of the following properties: (a) $T$ contains a radial path (which is necessarily unique). (b) $T \cong T_{n}$ for some $n>1$, where $T_{n}$ ( $n$ natural number) is the tree defined by $V\left(T_{n}\right)=$ $\{1, \cdots, n+8\}, E\left(T_{n}\right)=\{[i, i+1] ; i=1, \cdots, n+5\} \cup[4, n+7] \cup[n+$ $4, n+8]$, (the tree $T_{1}$ is defined in the Introduction)

(ii) $\mathfrak{A}(T)$ satisfies one of the following properties: (a) For every $\left[T_{i}\right] \in \mathfrak{R}(T), T_{i}$ contains a branch which is the path of length $r$. (b) There is $\left[T_{0}\right] \in \mathfrak{A}(T)$ such that every tree $T_{i},\left[T_{0}\right] \neq\left[T_{i}\right] \in \mathfrak{A}(T)$ contains a branch which is the path of length $r$, the tree $T_{0}$ itself contains a branch at one of its centers which is the path of length $r-1$.

Outline of proof. Let $T$ contain a radial path. Since $T$ is central, we can assume that $W(c, y)$ is a radial path $(d(y, T)=1)$. By asymmetry this is the only radial path in $T$. Furthermore: If $x \in V(T)-W(c, y), x \notin T^{\prime} \in A(T)$ then $T^{\prime}$ has a radial path. From this it follows, by the maximality of the elements of $A(T)$, that there is at most one $\left[T_{0}\right] \in \mathfrak{U}(T)$, such that $T_{0}$ has no branch which is the path of length $r$. It is now easy to conclude that either (iia) or (iib) holds. Conversely let $T$ have no radial path and suppose that $\mathfrak{V}(T)$ satisfies (iia) or (iib). We can conclude that $T \cong T_{n}$ for some $n>1$. We can prove first that every limb of $T$ is a radial limb and by a similar method to that in the proof of Proposition 2.2 we can prove $T \cong T_{n}$. The details are omitted.

Proposition 2.5. Let $n>1$. The tree $T_{n}$ see Lemma 2.2 is reconstructible from $\mathfrak{U}\left(T_{n}\right)$.

The proof is simple (using Proposition 2.3).

Proposition 2.6. If $T$ contains a radial path, then $T$ is reconstructible from $\mathfrak{A}(T)$.

Proof. Let $T^{\prime}$ contain the radial path (which is unique), and let $\mathfrak{U}\left(T^{\prime}\right)=\mathfrak{U}(T)$. Then by Lemma 2.2 and Proposition 2.5 we know that $T$ has the radial path. Assume that (iib) of the Lemma 2.2 holds; let $\left|T_{0}\right|=|T|-k$, then either diam $T_{0}=\operatorname{diam} T$ and $T \cong T^{\prime}$ follows easily, or diam $T_{0}<\operatorname{diam} T$. This case can occur if $T$ has only two 
radial branches at $c$. Since (iib) holds, we know that $R \neq \varnothing$, where $R$ is the union of all non-radial limbs at $c$. Applying Lemma 2.2 to $(R, c)$ we can determine both radial branches of $T$. It is easy to see also that $R$ is determined uniquely.

Case Lemma 2.2 (iia) can be handled similarly.

Now we can prove the main theorem for central trees:

Proposition 2.7. Let $T$ be central, then $T$ is reconstructible from $\mathfrak{N}(T)$.

Proof. By Propositions 2.1, 2.2, 2.5, 2.6 we can assume $T \not T_{1, k, m}$, $T \not T_{n}, T$ does not contain a radial path. Consider $\mathfrak{N}_{1}=\left\{\left[T_{i}\right] \in \mathfrak{A}(T)\right.$; $\left|T_{i}\right|+1=|T|, T_{i}$ is central\}.

We know (by the assumptions on $T$ ), that there is $x \in R$, where $R$ is a limb of the minimal cardinality, such that $T-x \in \mathfrak{R}_{1}$. In this tree $T-x$ we know all limbs except $R$. Let $R_{1}$ be a limb of the minimal cardinality among all limbs different from $R$. Let $\left(R_{1}^{\prime}, c\right)$ be a maximal root-asymmetric subtree of the limb $\left(R_{1}, c\right)$, which contains a peripheral point. If there is $T^{\prime \prime} \in \mathfrak{A}_{1}$, such that $T^{\prime}$ does not contain the limb $\left(R_{1}, c\right)$ and $T^{\prime}$ contains the limb $\left(R_{1}^{\prime}, c\right)$, then the branch $(R, c)$ is the only branch in $T^{\prime}$ which was still unknown. If there is no such tree $T^{\prime}$ then $(R, c) \cong\left(R_{1}^{\prime}, c\right)$.

To prove the main theorem for bicentral trees we could modify the proofs of the previous propositions. We use a different proof.

Proposition 2.8. Let $T$ be a bicentral tree. Then $T$ is reconstructible from $\mathfrak{Q}(T)$.

Proof. Let $T$ be a bicentral tree of the diameter $2 r+1$; by the Proposition 2.2 we can assume $T \nRightarrow T_{1, k, m}$. If $T$ contains a branch which is a path of length $r+1$, then for such a tree a statement similar to Lemma 2.2 holds and $T$ can be reconstructed from $\mathfrak{l}(T)$ in a similar manner to that used in Proposition 2.6. Assume that $T$ does not contain a branch of length $r+1$. Let us form the tree $T^{\sim}$ by the definition given in the proof of Corollary 1.2. As seen from the proof of the Proposition 2.7, $T^{\vee}$ is determined by all the trees in $\mathfrak{A}(T \smile)$ which have the same diameter as $T^{\searrow}$. Since for such trees the operation " $\vee$ " preserves isomorphism the proposition follows.

Thus we finally have: 
THEOREM 2.1. Every asymmetric tree is reconstructible from $A(T)$. Every asymmetric tree is reconstructible from $\mathfrak{X}(T)$, with the exception of $T_{1}$ and $T_{1,3,4}$.

\section{REFERENCES}

1. J. A. Bondy, On Kelly's congruence for trees, Proc. Cambridge Philos. Soc., 65 (1969), 1-11.

2. F. Harary and E. Palmer, The reconstruction of a tree from its maximal subtrees, Canadian J. Math., 18 (1966), 803-810

3. F. Harary, Graph Theory, Addison Wesley, Mass., 1969

4. P. J. Kelly, A congruence theorem for trees, Pacific J. Math., 7 (1957), 961-968.

5. B. Manvel, Reconstructing of trees, Canadian J. Math. (to appear).

6. G. Polya, Kombinatorische anzahlbestimmungen fur gruppen, graphen und chemische verbindungen, Acta Math., 68 (1937), 145-254.

Received May 4, 1970 and in revised form July 9, 1970. This paper was written while the author was supported by McMaster University, Hamilton, Ontario, Canada.

Charles University of Prague 


\title{
PACIFIC JOURNAL OF MATHEMATICS
}

\author{
EDITORS
}

\author{
H. SAMelson \\ Stanford University \\ Stanford, California 94305 \\ C. R. HoBBy \\ University of Washington \\ Seattle, Washington 98105
}

J. DugunduI

Department of Mathematics

University of Southern California

Los Angeles, California 90007

RICHARD ARENS

University of California

Los Angeles, California 90024

\section{ASSOCIATE EDITORS}

\author{
E. F. BeCKENBACH
}

B. H. NEUMANN

F. WoLF

K. YOSHIDA

\section{SUPPORTING INSTITUTIONS}

\author{
UNIVERSITY OF BRITISH COLUMBIA \\ CALIFORNIA INSTITUTE OF TECHNOLOGY \\ UNIVERSITY OF CALIFORNIA \\ MONTANA STATE UNIVERSITY \\ UNIVERSITY OF NEVADA \\ NEW MEXICO STATE UNIVERSITY \\ OREGON STATE UNIVERSITY \\ UNIVERSITY OF OREGON \\ OSAKA UNIVERSITY \\ UNIVERSITY OF SOUTHERN CALIFORNIA
}

\author{
STANFORD UNIVERSITY \\ UNIVERSITY OF TOKYO \\ UNIVERSITY OF UTAH \\ WASHINGTON STATE UNIVERSITY \\ UNIVERSITY OF WASHINGTON \\ AMERICAN MATHEMATICAL SOCIETY \\ CHEVRON RESEARCH CORPORATION \\ NAVAL WEAPONS CENTER
}

The Supporting Institutions listed above contribute to the cost of publication of this Journal, but they are not owners or publishers and have no responsibility for its content or policies.

Mathematical papers intended for publication in the Pacific Journal of Mathematics should be in typed form or offset-reproduced, (not dittoed), double spaced with large margins. Underline Greek letters in red, German in green, and script in blue. The first paragraph or two must be capable of being used separately as a synopsis of the entire paper. The editorial "we" must not be used in the synopsis, and items of the bibliography should not be cited there unless absolutely necessary, in which case they must be identified by author and Journal, rather than by item number. Manuscripts, in duplicate if possible, may be sent to any one of the four editors. Please classify according to the scheme of Math. Rev. Index to Vol. 39. All other communications to the editors should be addressed to the managing editor, Richard Arens, University of California, Los Angeles, California, 90024.

50 reprints are provided free for each article; additional copies may be obtained at cost in multiples of 50 .

The Pacific Journal of Mathematics is published monthly. Effective with Volume 16 the price per volume (3 numbers) is $\$ 8.00$; single issues, $\$ 3.00$. Special price for current issues to individual faculty members of supporting institutions and to individual members of the American Mathematical Society: $\$ 4.00$ per volume; single issues $\$ 1.50$. Back numbers are available.

Subscriptions, orders for back numbers, and changes of address should be sent to Pacific Journal of Mathematics, 103 Highland Boulevard, Berkeley, California, 94708.

PUBLISHED BY PACIFIC JOURNAL OF MATHEMATICS, A NON-PROFIT CORPORATION

Printed at Kokusai Bunken Insatsusha (International Academic Printing Co., Ltd.), 7-17, Fujimi 2-chome, Chiyoda-ku, Tokyo, Japan. 


\section{Pacific Journal of Mathematics}

\section{Vol. 37, No. $3 \quad$ March, 1971}

Mohammad Shafqat Ali and Marvin David Marcus, On the degree of the

minimal polynomial of a commutator operator ................ 561

Howard Anton and William J. Pervin, Integration on topological

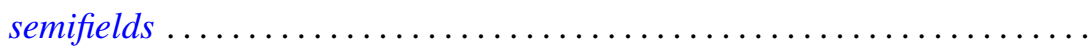

Martin Bartelt, Multipliers and operator algebras on bounded analytic

functions .................................... 575

Donald Earl Bennett, Aposyndetic properties of unicoherent continua ...... 585

James W. Bond, Lie algebras of genus one and genus two ............. 591

Mario Borelli, The cohomology of divisorial varieties ............... 617

Carlos R. Borges, How to recognize homeomorphisms and isometries ....... 625

J. C. Breckenridge, Burkill-Cesari integrals of quasi additive interval

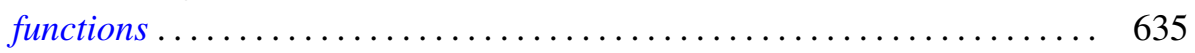

J. Csima, A class of counterexamples on permanents ................ 655

Carl Hanson Fitzgerald, Conformal mappings onto $\omega$-swirly domains . . . . . . 657

Newcomb Greenleaf, Analytic sheaves on Klein surfaces .............. 671

G. Goss and Giovanni Viglino, C-compact and functionally compact

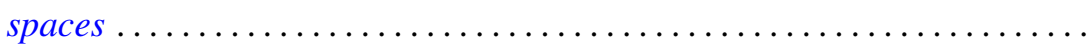

Charles Lemuel Hagopian, Arcwise connectivity of semi-aposyndetic plane

continua ..................................... 683

John Harris and Olga Higgins, Prime generators with parabolic limits ...

David Michael Henry, Stratifiable spaces, semi-stratifiable spaces, and their

relation through mappings .......................

Raymond D. Holmes, On contractive semigroups of mappings ........... 701

Joseph Edmund Kist and P. H. Maserick, BV-functions on semilattices ....... 711

Shûichirô Maeda, On point-free parallelism and Wilcox lattices ........... 725

Gary L. Musser, Linear semiprime $(p ; q)$ radicals ................. 749

William Charles Nemitz and Thomas Paul Whaley, Varieties of implicative

semilattices..................................... 759

Jaroslav Nešetřil, A congruence theorem for asymmetric trees ............ 771

Robert Anthony Nowlan, A study of $H$-spaces via left translations .......... 779

Gert Kjærgaard Pedersen, Atomic and diffuse functionals on a $C^{*}$-algebra ... 795

Tilak Raj Prabhakar, On the other set of the biorthogonal polynomials

suggested by the Laguerre polynomials...

801

Leland Edward Rogers, Mutually aposyndetic products of chainable

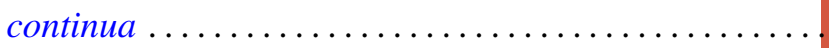

Frederick Stern, An estimate for Wiener integrals connected with squared

error in a Fourier series approximation.

Leonard Paul Sternbach, On k-shrinking and k-boundedly complete basic

sequences and quasi-reflexive spaces .................... 817

Pak-Ken Wong, Modular annihilator $A^{*}$-algebras ........ 